\title{
Confidence Intervals for Signal to Noise Ratio of a Poisson Distribution
}

\author{
Florence George and B.M. Golam Kibria \\ Department of Mathematics and Statistics, \\ Florida International University, Modesto A. Maidique Campus Miami, FL 33193, USA
}

Received 2012-08-02, Revised 2012-08-28; Accepted 2012-09-18

\begin{abstract}
The Poisson distribution is one of the most useful probability distributions to fit rare event data. Confidence interval for the SNR is an important issue among the researchers in image processing. This study considers several confidence intervals for the SNR of a Poisson distribution. Different confidence intervals available in literature are reviewed and compared based on the coverage probability and average width of the intervals. Since a theoretical comparison is not possible, a simulation study has been conducted to compare the performance of the interval estimators. Based on the simulation study we observed that most of our proposed interval estimators are performing well in the sense of attaining nominal size and they have been recommended for the researchers. Most of the proposed intervals except methods Wald, Waldz and bootstrap are performing well in the sense of attaining nominal size. The exact method performed the best followed by VSS, Wald B and Bayes in the sense of attaining nominal size and shorter width when the SNR is large.
\end{abstract}

Keywords: Average Width, Confidence Interval, Coverage Probability, Poisson Distribution, Signal to Noise Ratio, Simulation Study

\section{INTRODUCTION}

In some situations, the mean describes what is being measured, while the standard deviation represents noise and other interference. In these cases, the standard deviation is not important in itself, but only in comparison to the mean. This gives rise to the term: Signal-to-Noise Ratio (SNR), which is equal to the mean divided by the standard deviation. It is commonly used in image processing (for examples, Tania, 2008; Jitendra, 2009; John, 2007), where the SNR of an image is usually calculated as the ratio of the mean pixel value to the standard deviation of the pixel values over a given neighborhood. SNR measures how much signal has been corrupted by noise (McGibney and Smith, 1993). The population SNR is defined as a ratio of the population mean $(\mu)$ to the population standard deviation $(\sigma)$, i.e., $\operatorname{SNR}=\frac{\mu}{\sigma}$.
In real life examples like image processing, Signalto-Noise Ratio (SNR) describes the quality of a measurement. In Charge-Coupled Device (CCD) imaging, SNR refers to the relative magnitude of the signal compared to the uncertainty in that signal on a per-pixel basis. Specifically, it is the ratio of the measured signal to the overall measured noise at that pixel. High SNR is particularly important in applications requiring precise light measurement. The detected photons in a CCD camera or a photomultiplier tube follow a Poisson distribution, which is responsible for the Photon Noise and determines the Signal to Noise Ratio of the acquired image (Lee, 2009; Willkinson and Schut, 1998). Since the SNR of Poisson distribution has special interest in imaging, we will be discussing different methods of confidence interval for SNR of Poisson distribution here.

To test the significance of the SNR, a hypothesis test can be conducted and a confidence interval can be generated to reject or accept the null hypothesis. Confidence intervals associated with point estimates

Corresponding Author: Florence George, Department of Mathematics and Statistics, Florida International University, Modesto A. Maidique Campus Miami, FL 33193, USA 
provide more specific knowledge about the population characteristics than the p-values in the test of hypothesis (Visintainer and Tejani, 1998). The precision of a confidence interval can be determined through the width and coverage probability of the interval. Given constant coverage, as the width of the (1-a) $100 \%$ confidence interval decreases, the accuracy of the estimate increase (Kelley, 2007). The coverage level is the probability that the estimated interval will capture the true CV value (Banik and Kibria, 2010).

There are various methods available for estimating the confidence interval for a population $\mathrm{CV}$, such as, parametric, nonparametric, modified and bootstrapping (Banik and Kibria, 2010). The bootstrap approach is a non-parametric and computer-intensive tool used for estimating and making inferences about the parameters that was introduced by Efron (1979). It will be especially useful because, unlike other methods, this technique does not require any assumptions to be made about the underlying population of interest (Banik and Kibria, 2010). Therefore, bootstrapping technique can be applied for estimating or hypothesis testing to all situations. This method is implemented by simulating an original data set then randomly selecting data several times with replacement to estimate the parameter of a distribution. For more information on the confidence interval for the $\mathrm{CV}$, we refer Koopmans et al. (1964); Miller (1991); Sharma and Krishna (1994); McKay (1932); Vangel (1996) and very recently Banik and Kibria (2011) among others.

The literature on the confidence intervals for the SNR of a poisson distribution is very limited. The objective of this study is to propose some confidence interval estimators for SNR and find some good estimators for the practitioners. Six confidence intervals that already exist in literature for $\mathrm{CV}$ and they are considered for SNR by using the inverse relationship between CV and SNR. Additionally, we have made median and bootstrap modifications to several existing $\mathrm{CV}$ intervals in an attempt to improve the interval behavior. Since a theoretical comparison is not possible, a simulation study has been conducted to compare the performance of the interval estimators. Finally, based on the simulation results, the intervals with high coverage probability and smaller width were recommended for practitioners.

\section{MATERIALS AND METHODS}

Let $X_{1}, X_{2}, X_{3} \ldots X_{n}$ be an independently and identically distributed (iid) random sample of size $n$ from a poisson distribution with mean $\lambda$. Then the population SNR for Poisson distribution is $\sqrt{\lambda}$ and $\sqrt{\overline{\mathrm{x}}}$ would be the estimated value of the population $\mathrm{SNR}=\sqrt{\lambda}$. We want to find the $(1-\mathrm{a}) \quad 100 \%$ confidence intervals for the population SNR. We will review some interval estimators and propose some new interval estimators for SNR. Following 13 methods will be considered.

\subsection{Wald Method}

Historically, this is one of the first confidence intervals proposed for any parameter (Barker, 2002). The Wald confidence interval results from inverting the Wald test for $\lambda$. Using the asymptotic normality of the test statistic $Z=\frac{(\bar{X}-\lambda)}{\sqrt{\bar{X} / n}}$, the (1-a) $100 \%$ Confidence Interval (CI) for $\sqrt{\lambda}$ is $\left(\sqrt{\bar{X}-Z_{\alpha / 2} \sqrt{\frac{\bar{X}}{n}}}, \sqrt{\bar{X}+Z_{\alpha / 2} \sqrt{\frac{\bar{X}}{n}}}\right)$, where $\bar{X}$ denotes the sample mean and $Z_{c}$ denotes the 1-c quantile of the standard normal distribution.

\subsection{WaldB Method}

We estimate $\sqrt{\frac{\bar{X}}{n}}$ from bootstrap sample and obtained the following bootstrap version of Wald (named as WaldB) $(1-\alpha) 100 \%$ Confidence Interval (CI) for $\sqrt{\lambda}$

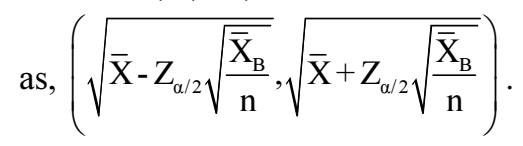

\subsection{WaldZ}

Wald confidence interval is based on the assumption that $\overline{\mathrm{X}}$ has an approximate normal distribution with mean $\lambda$ and variance $\frac{\lambda}{n}$. However, for small sample size, this assumption does not work well. Instead of using percentile points from Z-table, we propose to use percentile points from bootstrap samples. The proposed Wald lower and upper confidence intervals for $\sqrt{\lambda}$ are

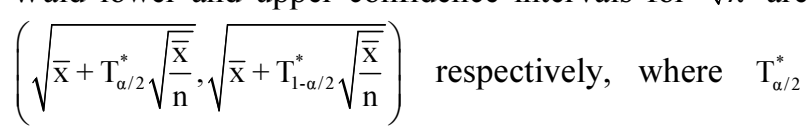
and $\mathrm{T}_{1-\alpha / 2}^{*}$ are the $(\alpha / 2)^{\text {th }}$ and $(1-\alpha / 2)^{\text {th }}$ sample quintiles of $\mathrm{T}_{\mathrm{i}}^{*}=\frac{\overline{\mathrm{x}}_{\mathrm{i}}^{*}-\overline{\mathrm{x}}_{\mathrm{B}}}{\hat{\sigma}_{\mathrm{B}}}$ where $\overline{\mathrm{x}}_{\mathrm{i}}^{*}$ is the ith bootstrap sample mean, $\hat{\sigma}_{\mathrm{B}}=\sqrt{\frac{1}{\mathrm{~B}-1} \sum_{\mathrm{i}=1}^{\mathrm{B}}\left(\overline{\mathrm{x}}_{\mathrm{i}}^{*}-\overline{\mathrm{x}}_{\mathrm{B}}\right)^{2}}$ and $\overline{\mathrm{x}}_{\mathrm{B}}=\frac{1}{\mathrm{~B}} \sum_{\mathrm{i}=1}^{\mathrm{B}} \overline{\mathrm{x}}_{\mathrm{i}}^{*}$.

\subsection{Wald with Continuity Correction (WCC)}

Since Wald interval uses a continuous distribution (normal) to approximate a discrete distribution (Poisson), 
a continuity correction might make this approximation more accurate (Barker, 2002). The Wald interval with continuity correction is given by:

$$
\left(\sqrt{\bar{X}-Z_{\alpha / 2} \sqrt{\frac{\bar{X}+0.5}{n}}}, \sqrt{\bar{X}+Z_{\alpha / 2} \sqrt{\frac{\bar{X}+0.5}{n}}}\right)
$$

\subsection{WecB Method}

We estimate $\sqrt{\frac{\bar{x}}{n}}$ from bootstrap sample and obtained the following bootstrap version of Wcc method (named as WccB) $(1-\alpha)$ 100\% Confidence Interval (CI) for $\sqrt{\lambda}$ as:

$$
\left(\sqrt{\bar{X}-Z_{\alpha / 2} \sqrt{\frac{\bar{X}_{B}+0.5}{n}}}, \sqrt{\bar{X}+Z_{\alpha / 2} \sqrt{\frac{\bar{X}_{B}+0.5}{n}}}\right)
$$

\subsection{WecZ}

Instead of using percentile points from Z-table, we propose to use percentile points from bootstrap samples. The proposed Wald with continuity correction the lower and upper confidence intervals for $\sqrt{\lambda}$ are, respectively:

$$
\left(\sqrt{\overline{\mathrm{x}}+\mathrm{T}_{\alpha / 2}^{*} \sqrt{\frac{\overline{\mathrm{x}}+0.5}{\mathrm{n}}}}, \sqrt{\overline{\mathrm{x}}+\mathrm{T}_{1-\alpha / 2}^{*} \sqrt{\frac{\overline{\mathrm{x}}+0.5}{\mathrm{n}}}}\right)
$$

\subsection{Scores (S)}

The Score confidence interval (Wilson, 1927) is on the basis of asymptotic normality of the test statistic $Z=\frac{(\bar{X}-\lambda)}{\sqrt{\lambda / n}}$. The end points of the $1-\alpha$ score confidence interval are the roots of the quadratic equation $Z_{\alpha / 2}^{2}=\left[\frac{(\bar{X}-\lambda)}{\sqrt{\lambda / n}}\right]^{2}$ and are given by:

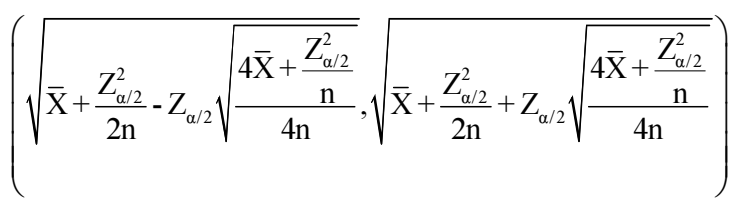

\subsection{Freeman and Turkey (FT)}

Freeman and Turkey (1950) showed that $\sqrt{\mathrm{n}}([\sqrt{\mathrm{X}}+\sqrt{\overline{\mathrm{X}}+1}]-[\sqrt{\lambda}+\sqrt{\lambda+1}])$ has an asymptotically standard normal distribution. Then following Barker (2002), the lower and upper confidence limits of $\sqrt{\lambda}$

are obtained as

$$
\left[\frac{\left(\sqrt{\bar{X}}+\sqrt{\bar{X}+1}-Z_{\alpha / 2} \frac{1}{\sqrt{n}}\right)^{2}-1}{2\left(\sqrt{\bar{X}}+\sqrt{\bar{X}+1}-Z_{\alpha / 2} \frac{1}{\sqrt{n}}\right)}\right] \text { and }
$$

$$
\left[\frac{\left(\sqrt{\bar{X}}+\sqrt{\bar{X}+1}+Z_{\alpha / 2} \frac{1}{\sqrt{\mathrm{n}}}\right)^{2}-1}{2\left(\sqrt{\bar{X}}+\sqrt{\bar{X}+1}+Z_{\alpha / 2} \frac{1}{\sqrt{\mathrm{n}}}\right)}\right]
$$

respectively. We need the restriction, $\left.\sqrt{\bar{X}}+\sqrt{\bar{X}+1}-Z_{\alpha / 2} \frac{1}{\sqrt{n}}\right)^{2}>1$ to be valid the above interval.

\subsection{Variance Stabilizing (VS)}

The variance stabilizing transformation (Bartlett, 1947) for a Poisson distribution is the square root transformation. The stabilized variance of the transformed variable is approximately 0.25 . Hence, the quantity $\frac{\sqrt{\mathrm{X}}-\sqrt{\lambda}}{\sqrt{1 / 4 n}}$ is asymptotically standard normal. This can be inverted into the interval:

$$
\left(\sqrt{\bar{X}+\frac{Z_{\alpha / 2}^{2}}{4 n}-Z_{\alpha / 2} \sqrt{\frac{\bar{X}}{n}}}, \sqrt{\bar{X}+\frac{Z_{\alpha / 2}^{2}}{4 n}+Z_{\alpha / 2} \sqrt{\frac{\bar{X}}{n}}}\right)
$$

\subsection{Recentered Variance Stabilizing (RVS)}

For any positive constant c, $\frac{\sqrt{\bar{X}+c}-\sqrt{\lambda+c}}{\sqrt{1 / 4 n}}$ is asymptotically standard normal? Anscombe (1948) showed that, as $\lambda$ tends to $\infty$, $\operatorname{var} \sqrt{(\overline{\mathrm{X}}+\mathrm{c})}=\frac{1+\frac{3-8 \mathrm{c}}{8 \lambda}}{4 \mathrm{n}}+\mathrm{o}(1 / \lambda)$. If $\mathrm{c}$ is $3 / 8$, the variance of $\sqrt{\bar{X}+0.5}$ is $\frac{1}{4 n}+O(1 / \theta)$. By inverting the expression $\frac{\sqrt{\mathrm{X}+\mathrm{c}}-\sqrt{\lambda+\mathrm{c}}}{\sqrt{1 / 4 \mathrm{n}}}$ we get the following confidence interval for $\sqrt{\lambda}$ :

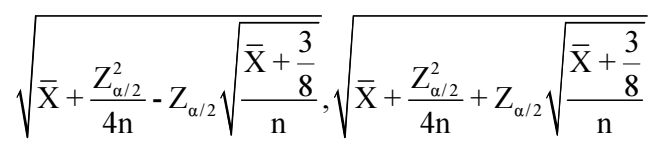

\subsection{Exact Method}

This method is based on an exact relationship between Poisson and Chi-square distribution and the end 
points of the exact interval are obtained by equating the tail sums of null Poisson probabilities to $\alpha / 2$ (Garwood 1936; Agresti and Coull, 1998). The exact method is designed to guarantee at least $100(1-\alpha) \%$ coverage. The lower and upper limits of exact intervals are:

$$
\sqrt{\frac{\chi_{\alpha / 2, \mathrm{df}}^{2}}{2 \mathrm{n}}}, \sqrt{\frac{\chi_{1-\alpha / 2, \mathrm{df}}^{2}}{2 \mathrm{n}}}
$$

Respectively, where $\mathrm{df}_{1}=2 * \sum \mathrm{X}$ and where, $\mathrm{df}_{2}=2\left(\sum \mathrm{X}+1\right)$.

\subsection{Bootstrap Approach}

Bootstrap is a commonly used computer-based nonparametric tool (introduced by Efron (1979; 1987), which requires no assumptions regarding the underlying population and can be applied to a variety of situations. An extensive array of different bootstrap methods is summarized as follows.

Let $\mathrm{X}^{(*)}=\mathrm{X}_{\mathrm{i} 1}^{(*)}, \mathrm{X}_{\mathrm{i} 2}^{(*)}, \ldots, \mathrm{X}_{\mathrm{in}}^{(*)}$, where the ith sample is denoted by $X_{(i)}^{*}$ for $I=1,2, B$ and $B$ is the number of bootstrap samples. The number of bootstrap samples is typically between 1000 and 2000; because, the accuracy of the confidence interval depends on the size of the samples (Efron and Tibshirani, 1993). The bootstrap estimate of $\mu$ is $\bar{x}_{B}$ of the $n$ estimates $\bar{x}_{i}^{*}$. Now, order the sample means of each bootstrap samples as follows: $\overline{\mathrm{x}}_{(1)}^{*} \leq \overline{\mathrm{x}}_{(2)}^{*} \leq \overline{\mathrm{x}}_{(3)}^{*} \leq \ldots \overline{\mathrm{x}}_{(\mathrm{B})}^{*}$. Then CI for $\sqrt{\lambda}$ is obtained as:

$$
\mathrm{L}=\sqrt{\overline{\mathrm{X}}_{[(\alpha / 2) \times \mathrm{B}]}^{*}} \text { and } \mathrm{U}=\sqrt{\overline{\mathrm{X}}_{[(1-(\alpha / 2)) \times \mathrm{B}]}^{*}}
$$

where, $(\alpha / 2) \times B$ and $(1-\alpha / 2) \times B$ quantiles of the bootstrap samples. This is known as non-parametric bootstrap confidence interval. In addition to these methods we want to propose the following two intervals.

\subsection{Bayes Approach}

The non-informative Jefferys prior plays a special role in the Bayesian analysis. The Jefferys prior for Poisson parameter $\lambda$ is proportional to $\lambda^{-1 / 2}$ which is improper and the posterior is Gamma $\left(\right.$ shape $=\sum X+\frac{1}{2}$, rate $\left.=n\right)($ Cai, 2005). Hence the lower limit and upper limit of confidence interval for $\sqrt{\lambda}$ are:

$$
\sqrt{\mathrm{G}_{\alpha / 2, \text { shape }=\sum \mathrm{X}+0.5, \text { rate }=\mathrm{n}}} \text { and } \sqrt{\mathrm{G}_{1-\alpha / 2, \text { shape }=\sum \mathrm{X}+0.5, \text { rate }=\mathrm{n}}}
$$

Respectively, where $\mathrm{G}$ represents the cdf of a gamma distribution. All of the above interval estimators are summarized in Table 1.

\subsection{Simulation Study}

In this study we considered 13 different confidence intervals for estimating the population SNR of a Poisson distribution. Since a theoretical comparison is not possible, a Monte-Carlo simulation is conducted using the $\mathrm{R}$ version 2.14.0 software to compare the performance of the interval estimators. The performance of the estimators was considered for various SNR values, confidence level and sample sizes.

\begin{tabular}{|c|c|c|}
\hline Method & Lower critical value & Upper critical value \\
\hline Wald & $\overline{\mathrm{X}}-\mathrm{Z}_{\alpha / 2} \sqrt{\frac{\overline{\mathrm{X}}}{\mathrm{n}}}$ & $\sqrt{\overline{\mathrm{X}}+Z_{\alpha / 2} \sqrt{\frac{\bar{X}}{n}}}$ \\
\hline WaldB & $\bar{X}-Z_{\alpha / 2} \sqrt{\frac{\bar{X}_{B}}{n}}$ & $+Z_{\alpha / 2} \sqrt{\frac{\bar{X}_{B}}{n}}$ \\
\hline Waldz & $+\mathrm{T}_{\alpha / 2}^{*} \sqrt{\frac{\bar{x}}{\mathrm{n}}}$ & $+\mathrm{T}_{1-\alpha / 2}^{*} \sqrt{\frac{\bar{x}}{n}}$ \\
\hline Wcc & $\overline{\mathrm{X}}-\mathrm{Z}_{\alpha / 2} \sqrt{\frac{\overline{\mathrm{X}}+0.5}{\mathrm{n}}}$ & $\sqrt{\bar{X}}+Z_{\alpha / 2} \sqrt{\frac{\bar{X}+0.5}{n}}$ \\
\hline WccB & $\overline{\mathrm{X}}-\mathrm{Z}_{\alpha / 2} \sqrt{\frac{\overline{\mathrm{X}}_{\mathrm{B}}+0.5}{\mathrm{n}}}$ & $\overline{\mathrm{X}}+\mathrm{Z}_{\alpha / 2} \sqrt{\frac{\overline{\mathrm{X}}_{\mathrm{B}}+0.5}{\mathrm{n}}}$ \\
\hline Wccz & $\sqrt{\mathrm{x}}+\mathrm{T}_{\alpha / 2}^{*} \sqrt{\frac{\overline{\mathrm{x}}+0.5}{\mathrm{n}}}$ & $\sqrt{\overline{\mathrm{x}}+\mathrm{T}_{1-\alpha \cdot 2}^{*} \sqrt{\frac{\overline{\mathrm{x}+0.5}}{\mathrm{n}}}}$ \\
\hline S & $\sqrt{\bar{X}}+\frac{Z_{a / 2}^{2}}{2 \mathrm{n}}-Z_{a / 2} \sqrt{\frac{4 \bar{X}+\frac{Z_{a / 2}^{2}}{n}}{4 \mathrm{n}}}$ & $\sqrt{\overline{\mathrm{X}}+\frac{Z_{\alpha / 2}^{2}}{2 \mathrm{n}}+Z_{\alpha / 2} \sqrt{\frac{4 \overline{\mathrm{X}}+\frac{Z_{\alpha / 2}^{2}}{\mathrm{n}}}{4 \mathrm{n}}}}$ \\
\hline FT & $\frac{\left(\sqrt{\bar{X}}+\sqrt{\bar{X}+1}-Z_{\alpha / 2} \frac{1}{\sqrt{n}}\right)^{2}-1}{2\left(\sqrt{\bar{X}}+\sqrt{\bar{X}+1}-Z_{\alpha / 2} \frac{1}{\sqrt{n}}\right)}$ & {$\left[\frac{\left(\sqrt{\overline{\mathrm{X}}}+\sqrt{\overline{\mathrm{X}}+1}+Z_{\alpha / 2} \frac{1}{\sqrt{\mathrm{n}}}\right)^{2}-1}{2\left(\sqrt{\mathrm{X}}+\sqrt{\mathrm{X}+1}+Z_{\alpha / 2} \frac{1}{\sqrt{\mathrm{n}}}\right)}\right]$} \\
\hline VSS & $\sqrt{\bar{X}}+\frac{Z_{\alpha / 2}^{2}}{4 n}-Z_{\alpha / 2} \sqrt{\frac{\bar{X}}{n}}$ & $\sqrt{\overline{\mathrm{X}}+\frac{\mathrm{Z}_{\alpha / 2}^{2}}{4 \mathrm{n}}+\mathrm{Z}_{\alpha / 2} \sqrt{\frac{\overline{\mathrm{X}}}{\mathrm{n}}}}$ \\
\hline RVS & $\sqrt{\overline{\mathrm{X}}+\frac{Z_{\alpha / 2}^{2}}{4 \mathrm{n}}-Z_{\alpha / 2} \sqrt{\frac{\overline{\mathrm{X}}+\frac{3}{8}}{\mathrm{n}}}}$ & $\sqrt{\bar{X}}+\frac{Z_{\alpha / 2}^{2}}{4 n}+Z_{\alpha / 2} \sqrt{\frac{\bar{X}+\frac{3}{8}}{n}}$ \\
\hline Exact & $\sqrt{\frac{\chi_{\alpha / 2, \mathrm{df}_{1}}^{2}}{2 \mathrm{n}}}$ & $\sqrt{\frac{\chi_{1-\alpha / 2, \mathrm{df}_{2}}^{2}}{2 \mathrm{n}}}$ \\
\hline Boot & $\sqrt{\overline{\mathrm{X}}_{[(\alpha / 2) \mathrm{xB}]}^{*}}$ & $\sqrt{\overline{\mathrm{x}}_{[(1-(\alpha / 2)) \mathrm{xB}]}^{*}}$ \\
\hline Bayes & $\sqrt{\mathrm{G}_{\alpha / 2, \text { shape }}=\sum \mathrm{X}+0.5, \text { rate }=\mathrm{n}}$ & $\sqrt{\mathrm{G}_{1-\alpha / 2, \text { shape }}=\sum \mathrm{X}+0.5, \text { rate }=\mathrm{n}}$ \\
\hline
\end{tabular}

Table 1. Lower and upper critical values of poisson SNR based on proposed methods 
To see the behavior of small and large sample sizes, we used $\mathrm{n}=5,10,15,20,30,50,100$. A random sample was generated with specific parameters $(\lambda=1.0,2.0,5.0$ and 10) from Poisson distribution Eq. 1:

$$
p(x)=\frac{e^{-\lambda} \lambda^{x}}{x !}, x=0,1,2, \ldots
$$

If the data are from a symmetric distribution (or $n$ is large), the coverage probability will be exact or close to $1-\alpha$. So the coverage probability would be a useful criterion for evaluating the confidence interval. Another criterion is the width of the confidence interval. A smaller width gives a more reliable confidence interval.
When the coverage probabilities for different methods are the same, a smaller width indicates that the method is appropriate for the specific sample. In the bootstrap calculations we used 1000 bootstrap samples (Efron, $1979 ;$ 1987). The below and above rates of a confidence interval is the fraction out of 5,000 samples that resulted in an interval that lies entirely above and below the true value of the population mean. The coverage probability is found as the sum of the lower rate and upper rate and then subtracted from total probability 1 . The simulated results for $\lambda=1.0,2.0,5.0$ and 10.0 are presented in Table 1-5 for $90 \%$ confidence intervals and in Table 6-9 for $95 \%$ confidence intervals.

Table 2. The coverage probabilities and average widths for lambda $=1$ and $1-\alpha=0.90$

\begin{tabular}{|c|c|c|c|c|c|c|c|c|c|c|c|c|c|c|}
\hline $\mathrm{N}$ & & Wald & WaldB & Waldz & Wcc & $\mathrm{WccB}$ & Wccz & $\mathrm{S}$ & FT & VSS & RVS & Exact & Boot & Bayes \\
\hline \multirow[t]{4}{*}{5} & Cover & 0.861 & 0.861 & 0.749 & 0.962 & 0.961 & 0.875 & 0.903 & 0.955 & 0.939 & 0.939 & 0.939 & 0.665 & 0.903 \\
\hline & Lower & 0.012 & 0.013 & 0.080 & 0.005 & 0.006 & 0.045 & 0.065 & 0.012 & 0.028 & 0.028 & 0.028 & 0.115 & 0.065 \\
\hline & Upper & 0.127 & 0.127 & 0.171 & 0.033 & 0.033 & 0.080 & 0.033 & 0.033 & 0.033 & 0.033 & 0.033 & 0.220 & 0.033 \\
\hline & Width & 0.807 & 0.807 & 0.673 & 0.960 & 0.960 & 0.784 & 0.729 & 0.881 & 0.729 & 0.906 & 0.819 & 0.736 & 0.718 \\
\hline \multirow[t]{4}{*}{10} & Cover & 0.903 & 0.877 & 0.824 & 0.955 & 0.956 & 0.903 & 0.920 & 0.940 & 0.883 & 0.940 & 0.920 & 0.778 & 0.883 \\
\hline & Lower & 0.032 & 0.031 & 0.058 & 0.017 & 0.016 & 0.045 & 0.053 & 0.032 & 0.053 & 0.032 & 0.053 & 0.072 & 0.053 \\
\hline & Upper & 0.065 & 0.092 & 0.117 & 0.028 & 0.028 & 0.052 & 0.028 & 0.028 & 0.065 & 0.028 & 0.028 & 0.150 & 0.065 \\
\hline & Width & 0.544 & 0.544 & 0.491 & 0.689 & 0.689 & 0.619 & 0.520 & 0.620 & 0.520 & 0.633 & 0.567 & 0.491 & 0.516 \\
\hline \multirow{4}{*}{15} & Cover & 0.896 & 0.894 & 0.855 & 0.950 & 0.950 & 0.926 & 0.906 & 0.941 & 0.896 & 0.941 & 0.930 & 0.822 & 0.906 \\
\hline & Lower & 0.029 & 0.028 & 0.045 & 0.010 & 0.010 & 0.027 & 0.053 & 0.019 & 0.029 & 0.019 & 0.029 & 0.059 & 0.053 \\
\hline & Upper & 0.075 & 0.077 & 0.099 & 0.041 & 0.041 & 0.048 & 0.041 & 0.041 & 0.075 & 0.041 & 0.041 & 0.118 & 0.041 \\
\hline & Width & 0.436 & 0.436 & 0.408 & 0.550 & 0.551 & 0.512 & 0.425 & 0.503 & 0.425 & 0.509 & 0.457 & 0.408 & 0.423 \\
\hline \multirow[t]{4}{*}{20} & Cover & 0.903 & 0.900 & 0.870 & 0.951 & 0.951 & 0.940 & 0.911 & 0.944 & 0.903 & 0.944 & 0.931 & 0.845 & 0.911 \\
\hline & Lower & 0.034 & 0.032 & 0.047 & 0.014 & 0.014 & 0.023 & 0.054 & 0.021 & 0.034 & 0.021 & 0.034 & 0.059 & 0.054 \\
\hline & Upper & 0.063 & 0.068 & 0.083 & 0.035 & 0.035 & 0.037 & 0.035 & 0.035 & 0.063 & 0.035 & 0.035 & 0.096 & 0.035 \\
\hline & Width & 0.375 & 0.375 & 0.358 & 0.468 & 0.468 & 0.447 & 0.368 & 0.434 & 0.368 & 0.438 & 0.392 & 0.358 & 0.367 \\
\hline \multirow[t]{4}{*}{30} & Cover & 0.885 & 0.885 & 0.871 & 0.947 & 0.947 & 0.936 & 0.914 & 0.939 & 0.896 & 0.939 & 0.914 & 0.851 & 0.896 \\
\hline & Lower & 0.031 & 0.031 & 0.047 & 0.014 & 0.014 & 0.023 & 0.046 & 0.021 & 0.046 & 0.021 & 0.046 & 0.053 & 0.046 \\
\hline & Upper & 0.084 & 0.084 & 0.082 & 0.039 & 0.039 & 0.041 & 0.039 & 0.039 & 0.058 & 0.039 & 0.039 & 0.096 & 0.058 \\
\hline & Width & 0.304 & 0.304 & 0.293 & 0.377 & 0.377 & 0.363 & 0.300 & 0.354 & 0.300 & 0.356 & 0.316 & 0.293 & 0.300 \\
\hline \multirow[t]{4}{*}{50} & Cover & 0.904 & 0.900 & 0.884 & 0.945 & 0.950 & 0.945 & 0.894 & 0.939 & 0.892 & 0.939 & 0.906 & 0.872 & 0.894 \\
\hline & Lower & 0.032 & 0.036 & 0.049 & 0.018 & 0.018 & 0.022 & 0.058 & 0.024 & 0.045 & 0.024 & 0.045 & $0.05 \overline{3}$ & 0.058 \\
\hline & Upper & 0.064 & 0.064 & 0.067 & 0.037 & 0.033 & 0.032 & 0.049 & 0.037 & 0.064 & 0.037 & 0.049 & 0.075 & 0.049 \\
\hline & Width & 0.234 & 0.234 & 0.229 & 0.289 & 0.289 & 0.282 & 0.233 & 0.273 & 0.233 & 0.274 & 0.242 & 0.229 & 0.232 \\
\hline \multirow[t]{4}{*}{100} & Cover & 0.906 & 0.906 & 0.898 & 0.955 & 0.959 & 0.953 & 0.906 & 0.952 & 0.906 & 0.948 & 0.914 & 0.892 & 0.906 \\
\hline & Lower & 0.041 & 0.037 & 0.044 & 0.019 & 0.018 & 0.022 & 0.049 & 0.022 & 0.041 & 0.026 & 0.041 & 0.048 & 0.049 \\
\hline & Upper & 0.054 & 0.057 & 0.058 & 0.026 & 0.023 & 0.025 & 0.045 & 0.026 & 0.054 & 0.026 & 0.045 & 0.060 & 0.045 \\
\hline & Width & 0.165 & 0.165 & 0.163 & 0.203 & 0.203 & 0.200 & 0.164 & 0.193 & 0.164 & 0.193 & 0.169 & 0.163 & 0.164 \\
\hline $\mathrm{N}$ & & \multicolumn{13}{|c|}{ Table 3. The coverage probabilities and average widths for lambda $=2$ and $1-\alpha=0.90$} \\
\hline \multirow[t]{4}{*}{5} & Cover & 0.901 & 0.881 & 0.774 & 0.916 & 0.916 & 0.834 & 0.918 & 0.940 & 0.880 & 0.901 & 0.918 & 0.722 & 0.880 \\
\hline & Lower & 0.030 & 0.030 & 0.085 & 0.016 & 0.016 & 0.061 & 0.052 & 0.030 & 0.052 & 0.030 & 0.052 & 0.107 & 0.052 \\
\hline & Upper & 0.068 & 0.089 & 0.141 & 0.068 & 0.068 & 0.105 & 0.030 & 0.030 & 0.068 & 0.068 & 0.030 & 0.171 & 0.068 \\
\hline & Width & 0.769 & 0.769 & 0.649 & 0.877 & 0.877 & 0.734 & 0.735 & 0.820 & 0.735 & 0.818 & 0.802 & 0.658 & 0.730 \\
\hline \multirow[t]{4}{*}{10} & Cover & 0.906 & 0.903 & 0.840 & 0.920 & 0.932 & 0.882 & 0.918 & 0.934 & 0.906 & 0.934 & 0.934 & 0.812 & 0.918 \\
\hline & Lower & 0.033 & 0.032 & 0.061 & 0.020 & 0.020 & 0.048 & 0.049 & 0.033 & 0.033 & 0.033 & 0.033 & 0.071 & 0.049 \\
\hline & Upper & 0.061 & 0.065 & 0.099 & 0.061 & 0.049 & 0.071 & 0.032 & 0.032 & 0.061 & 0.032 & 0.032 & 0.116 & 0.032 \\
\hline & Width & 0.530 & 0.530 & 0.484 & 0.599 & 0.599 & 0.546 & 0.520 & 0.576 & 0.520 & 0.571 & 0.554 & 0.484 & 0.518 \\
\hline \multirow[t]{4}{*}{15} & Cover & 0.888 & 0.889 & 0.863 & 0.923 & 0.924 & 0.899 & 0.921 & 0.933 & 0.902 & 0.933 & 0.921 & 0.841 & 0.902 \\
\hline & Lower & 0.031 & 0.032 & 0.053 & 0.023 & 0.023 & 0.041 & 0.044 & 0.031 & 0.044 & 0.031 & 0.044 & 0.062 & 0.044 \\
\hline & Upper & 0.081 & 0.079 & 0.084 & 0.054 & 0.053 & 0.060 & 0.036 & 0.036 & 0.054 & 0.036 & 0.036 & 0.096 & 0.054 \\
\hline & Width & 0.430 & 0.430 & 0.405 & 0.484 & 0.484 & 0.455 & 0.425 & 0.470 & 0.425 & 0.465 & 0.447 & 0.405 & 0.424 \\
\hline \multirow[t]{4}{*}{20} & Cover & 0.896 & 0.894 & 0.868 & 0.934 & 0.930 & 0.906 & 0.898 & 0.924 & 0.896 & 0.924 & 0.913 & 0.850 & 0.898 \\
\hline & Lower & 0.043 & 0.040 & 0.054 & 0.022 & 0.026 & 0.042 & 0.058 & 0.032 & 0.043 & 0.032 & 0.043 & 0.062 & 0.058 \\
\hline & Upper & 0.061 & 0.065 & 0.077 & 0.044 & 0.044 & 0.052 & 0.044 & 0.044 & 0.061 & $0.04 \overline{4}$ & 0.044 & 0.088 & 0.044 \\
\hline & Width & 0.371 & 0.371 & 0.354 & 0.417 & 0.417 & 0.398 & 0.368 & 0.406 & 0.368 & 0.402 & 0.385 & 0.354 & 0.367 \\
\hline \multirow[t]{4}{*}{30} & Cover & 0.907 & 0.904 & 0.885 & 0.929 & 0.933 & 0.923 & 0.897 & 0.936 & 0.913 & 0.936 & 0.913 & 0.872 & 0.913 \\
\hline & Lower & 0.027 & 0.030 & 0.044 & 0.021 & 0.021 & 0.027 & 0.054 & 0.027 & 0.037 & 0.027 & 0.037 & 0.051 & 0.037 \\
\hline & Upper & 0.066 & 0.066 & 0.070 & 0.050 & 0.046 & 0.050 & 0.050 & 0.037 & 0.050 & 0.037 & 0.050 & 0.077 & 0.050 \\
\hline & Width & 0.302 & 0.302 & 0.292 & 0.339 & 0.339 & 0.328 & 0.300 & 0.331 & 0.300 & 0.328 & 0.312 & 0.292 & 0.300 \\
\hline 50 & Cover & 0.903 & 0.902 & 0.894 & 0.937 & 0.935 & 0.926 & 0.904 & 0.931 & 0.903 & 0.931 & 0.913 & 0.884 & 0.904 \\
\hline & Lower & 0.040 & 0.036 & 0.043 & 0.025 & 0.024 & 0.029 & 0.048 & 0.031 & 0.040 & 0.031 & 0.040 & 0.050 & 0.048 \\
\hline & Upper & 0.057 & 0.061 & 0.062 & 0.038 & 0.041 & 0.044 & 0.048 & 0.038 & 0.057 & 0.038 & 0.048 & 0.067 & 0.048 \\
\hline & Width & 0.233 & 0.233 & 0.229 & 0.261 & 0.261 & 0.256 & 0.233 & 0.256 & 0.233 & 0.254 & 0.240 & 0.229 & 0.232 \\
\hline 100 & Cover & 0.895 & 0.898 & 0.893 & 0.931 & 0.932 & 0.928 & 0.904 & 0.926 & 0.896 & 0.926 & 0.910 & 0.887 & 0.896 \\
\hline & Lower & 0.044 & 0.044 & 0.050 & 0.030 & 0.031 & 0.032 & 0.051 & 0.034 & 0.051 & 0.034 & 0.044 & 0.053 & 0.051 \\
\hline & Upper & 0.061 & 0.058 & 0.057 & 0.040 & 0.038 & 0.040 & 0.046 & 0.040 & 0.053 & 0.040 & 0.046 & 0.060 & 0.053 \\
\hline & Width & 0.165 & 0.165 & 0.163 & 0.184 & 0.184 & 0.182 & 0.164 & 0.181 & 0.164 & 0.179 & 0.168 & 0.163 & 0.164 \\
\hline
\end{tabular}


Florence George and B.M. Golam Kibria / American Journal of Biostatistics 2 (2) (2011) 44-55

Table 4. The estimated coverage probabilities and average widths for lambda $=5$ and confidence coefficient 0.90

\begin{tabular}{|c|c|c|c|c|c|c|c|c|c|c|c|c|c|c|}
\hline$\overline{\mathrm{N}}$ & & Wald & WaldB & Waldz & Wcc & WccB & Wccz & $\mathrm{S}$ & FT & VSS & RVS & Exact & Boot & Bayes \\
\hline \multirow[t]{4}{*}{5} & Cover & 0.878 & 0.889 & 0.786 & 0.918 & 0.914 & 0.822 & 0.910 & 0.905 & 0.889 & 0.905 & 0.910 & 0.749 & 0.889 \\
\hline & Lower & .035 & 0.035 & 0.091 & 0.023 & 0.026 & 0.075 & 0.052 & 0.035 & 0.052 & 0.035 & .052 & 0.106 & 0.052 \\
\hline & Upper & 0.087 & 0.076 & 0.124 & 0.059 & 0.059 & 0.103 & 0.038 & 0.059 & 0.059 & 0.059 & .038 & 0.145 & 0.05 \\
\hline & Width & .747 & .747 & .633 & 0.786 & 0.786 & 0.666 & .736 & 0.771 & .736 & 0.765 & 0.779 & .633 & 0.734 \\
\hline \multirow[t]{4}{*}{10} & Cover & 398 & 894 & 845 & 0.898 & 0.904 & 0.865 & 891 & 0.903 & .888 & 903 & 903 & 829 & .891 \\
\hline & Lower & .034 & 0.038 & .065 & 0.034 & 0.034 & 0.056 & 0.057 & 0.044 & 0.044 & 0.044 & 0.044 & 0.071 & 0.057 \\
\hline & Upper & 0.067 & 0.068 & .090 & 0.067 & 0.062 & 0.079 & .052 & 0.052 & 0.067 & 0.052 & .052 & 0.100 & 0.052 \\
\hline & Width & .524 & 0.524 & 484 & 0.550 & 0.550 & 0.508 & 520 & 0.545 & .520 & 0.540 & .542 & .484 & $0.51 \mathrm{C}$ \\
\hline \multirow[t]{4}{*}{15} & Cover & 36 & 895 & 53 & 0.910 & 0 & .870 & 07 & 0.918 & .892 & 0.918 & 18 & 44 & 0.892 \\
\hline & Lower & 037 & 0.036 & .059 & 0.030 & 0.030 & 0.051 & .047 & 0.037 & 0.047 & 0.037 & .037 & .064 & 0.047 \\
\hline & oper & 077 & 0.069 & .088 & 0.060 & .060 & 0.079 & .046 & 0.046 & 0.060 & 0.046 & 0.046 & 0.093 & 0.060 \\
\hline & Width & 427 & 0.427 & 401 & 0.448 & & 0.421 & 0.425 & 0.444 & 0.425 & 0.441 & 0.439 & 0.401 & 0.424 \\
\hline \multirow[t]{4}{*}{20} & Cover & 8 & 893 & 65 & 0.905 & & 0.882 & .899 & 0.909 & 0.898 & 0.909 & 09 & 52 & 0.899 \\
\hline & wer & .042 & .040 & .054 & 0.034 & 0.034 & 0.048 & .052 & 0.042 & 0.042 & 0.042 & 42 & 600 & 0.052 \\
\hline & per & 060 & .067 & 082 & 0.060 & & 0.070 & .049 & 0.049 & 0.060 & 0.049 & 0.049 & 0.089 & 0.04 \\
\hline & Width & 369 & 0.369 & 52 & 0.387 & & 0.369 & 68 & 0. & 68 & 82 & 79 & 52 & 0.36 \\
\hline \multirow[t]{4}{*}{30} & Cover & & 01 & & 0.915 & & 0 . & 10 & 0.918 & 0.901 & 0.918 & 10 & 71 & 0.90 \\
\hline & pwer & .041 & .042 & .056 & 0.035 & 36 & 0.049 & .049 & 0.041 & 0.049 & 0.041 & 0.049 & 0.061 & 0.049 \\
\hline & per & & 057 & 063 & 0.050 & & 0.055 & & 0.041 & .050 & 0.041 & & 068 & 0.050 \\
\hline & Width & 0.301 & 0.301 & 0.292 & 0.316 & & 0.306 & .300 & 0.314 & 0.300 & 0.311 & 0.308 & 0.292 & 0.30 \\
\hline \multirow[t]{4}{*}{50} & Cover & 902 & .901 & 0.892 & 0.914 & & 0.907 & .909 & 0.915 & 0.902 & .915 & 09 & 886 & 0.902 \\
\hline & & & & & & & & & & & & & & 0.052 \\
\hline & er & & 52 & & & & & & & & & & 058 & 0.046 \\
\hline & Width & 0.233 & 0.233 & 0.229 & 0.244 & 0.244 & 0.240 & 0.233 & 0.243 & 0.233 & 0.241 & 0.237 & 0.229 & 0.23 \\
\hline \multirow[t]{4}{*}{100} & Cover & .899 & 0.899 & 0.889 & 0.912 & 0.912 & 0.905 & 0.898 & 0.912 & 0.900 & 0.908 & 0.900 & 0.885 & 0.900 \\
\hline & & & & & & & & & 0.043 & & & & 54 & 0.051 \\
\hline & & & & & & & & & & & & & .060 & 0.049 \\
\hline & Width & 0.165 & 0.165 & 0.163 & 0.173 & 0.173 & 0.171 & 0.164 & 0.172 & 0.164 & 0.171 & 0.167 & 0.163 & 0.164 \\
\hline
\end{tabular}

Table 5. The estimated coverage probabilities and average widths for lambda $=10$ and confidence coefficient 0.90

\begin{tabular}{|c|c|c|c|c|c|c|c|c|c|c|c|c|c|c|}
\hline $\mathrm{N}$ & & Wald & WaldB & Waldz & Wcc & WccB & Wccz & $\mathrm{S}$ & FT & VSS & RVS & Exact & Boot & Bayes \\
\hline \multirow[t]{4}{*}{5} & Cover & 0.898 & 0.894 & 0.780 & 0.898 & 0.898 & 0.799 & 0.890 & 0.902 & 0.887 & 0.902 & 902 & 0.759 & 0.890 \\
\hline & Lower & 037 & 041 & 097 & 0.037 & 0.038 & 089 & 0.060 & .048 & 0.048 & 0.048 & 48 & .108 & .060 \\
\hline & Upper & 065 & 65 & 123 & .065 & 0.064 & 112 & 050 & 050 & .065 & 0.050 & 550 & & .050 \\
\hline & Width & .741 & 0.741 & 0.627 & 0.760 & 0.760 & .643 & 0.736 & 0.754 & 0.736 & 0.750 & .766 & .627 & 0.735 \\
\hline \multirow[t]{4}{*}{10} & Cover & 0.902 & 0.900 & 0.844 & 0.912 & 0.912 & .854 & 0.906 & 0.914 & 0.902 & 0.914 & .914 & 0.833 & 0.906 \\
\hline & Lower & 0.042 & 0.039 & 0066 & 0.032 & 0.034 & 062 & 0.049 & .042 & .042 & 0.042 & & .070 & 0.049 \\
\hline & Upper & 0 & & & 6 & & 84 & 45 & & & & & 97 & 045 \\
\hline & Width & 522 & 0.522 & 0.479 & 535 & 0.535 & 491 & 520 & .533 & 0.520 & 0. & 36 & 79 & .520 \\
\hline \multirow[t]{4}{*}{15} & Cover & 0.892 & 0.893 & 0.860 & 0.907 & 0.902 & .870 & 0.903 & 0.900 & 0.893 & 0.900 & 03 & & 0.893 \\
\hline & Lower & 0.041 & 0.041 & 0.060 & 0.034 & 0.037 & .056 & 0.048 & 0.041 & & & & & 0.048 \\
\hline & & 067 & & & 0.058 & & 74 & 49 & & & & & & 0.058 \\
\hline & Width & 0.426 & & & 6 & 0.4 & & 25 & & & & & & 125 \\
\hline \multirow[t]{4}{*}{20} & Cover & 0.900 & 0.904 & 4 & 0.914 & 0.911 & 83 & 0.909 & 0.916 & 0.901 & 0.916 & 16 & 67 & 0.901 \\
\hline & Lower & .041 & 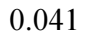 & & 0.035 & 0.038 & 54 & 049 & & & & & 62 & 0.049 \\
\hline & Upper & .058 & 0.055 & 68 & 0.051 & 0.051 & .063 & 0.042 & 0.042 & 0.051 & & & .072 & 0.051 \\
\hline & & & & & 0.378 & & & & & & & & & 0.368 \\
\hline \multirow[t]{4}{*}{30} & Cover & 0 & & & 0.900 & 0.903 & 35 & 94 & 02 & & & & & 0.894 \\
\hline & Lower & 051 & & & 0.046 & 0.046 & 56 & 59 & & & & & 63 & 0.059 \\
\hline & Upper & .054 & & 4 & 0.054 & 0.051 & 0.059 & 0.047 & & 0.054 & & & .066 & 0.047 \\
\hline & Width & 0.301 & 0.301 & 0.292 & 0.308 & 0.308 & 0.299 & 0.300 & 0.307 & 0.300 & 0.306 & & .292 & 0.300 \\
\hline \multirow[t]{4}{*}{50} & Cover & 0900 & & & 0.910 & & & 0.896 & & & & & & 0.901 \\
\hline & I ower & & & & & & & & & & & & & \\
\hline & & & & & & & & & & & & & 62 & 0.049 \\
\hline & Width & 0.233 & & 0.228 & 0.239 & 0.239 & 0.234 & 0.233 & 0.238 & 0.233 & 0.237 & & .228 & 0.233 \\
\hline \multirow[t]{4}{*}{100} & Cover & 0.897 & 0.898 & 0.892 & 0.904 & 0.906 & 0.900 & 0.900 & 0.903 & 0.897 & 0.903 & 0.900 & 0.891 & 0.897 \\
\hline & Lower & & & & & & & 2 & & & & & & 0.052 \\
\hline & & & & & & & & & & & & & 53 & \\
\hline & Width & 0.165 & 0.165 & 0.163 & 0.169 & 0.169 & 0.167 & 0.164 & 0.168 & 0.164 & 0.168 & 0.166 & 0.163 & 0.164 \\
\hline
\end{tabular}


Florence George and B.M. Golam Kibria / American Journal of Biostatistics 2 (2) (2011) 44-55

Table 6. The estimated coverage probabilities and average widths for lambda $=1$ and confidence coefficient 0.95

\begin{tabular}{|c|c|c|c|c|c|c|c|c|c|c|c|c|c|c|}
\hline$\overline{\mathrm{N}}$ & & Wald & WaldB & Naldz & Wcc & WccB & $\mathrm{Wccz}$ & $\mathrm{S}$ & FT & VSS & RVS & Exact & Boot & Baye \\
\hline \multirow[t]{4}{*}{5} & Cover & 0.868 & 0.868 & 0.761 & 0.966 & 0.966 & .880 & 0.972 & 0.995 & 0.955 & 0.995 & 0.988 & 0.681 & 0.939 \\
\hline & ower & 005 & 005 & 070 & 0.002 & 002 & 041 & 028 & 005 & 012 & 0.005 & 012 & 101 & .028 \\
\hline & Upper & .127 & 127 & .168 & 0.033 & 0.033 & 079 & 0.000 & 0.000 & 0.033 & 0.000 & .000 & .218 & .033 \\
\hline & Width & 1.003 & 003 & .757 & .166 & 1.166 & 907 & 0.869 & .051 & .869 & 1.059 & .952 & 13 & 0.852 \\
\hline \multirow[t]{4}{*}{10} & Cover & 0.925 & 0.925 & 0.881 & 0.968 & 0.969 & .945 & 0.960 & 0.981 & .955 & 0.981 & .974 & .845 & .940 \\
\hline & wer & 0 & & 5 & 04 & 0.004 & 22 & .032 & 10 & 17 & 0.010 & 17 & 18 & .032 \\
\hline & Upper & 065 & 65 & 85 & 0.028 & 0.027 & 34 & 0.009 & .009 & 28 & 0.009 & 009 & & .028 \\
\hline & Width & 664 & 0.664 & 0.589 & 0.845 & 0.846 & .736 & 0.620 & 0.741 & 0.620 & 0.758 & .665 & 0.593 & 0.614 \\
\hline \multirow[t]{4}{*}{15} & Cover & 916 & 0.921 & 0.907 & 0.978 & 0.978 & 961 & 0.952 & 0.972 & 0.941 & 0.972 & .963 & & 0.963 \\
\hline & & 0 & & 0.024 & 0.003 & 0.003 & 013 & 0.029 & 0.010 & & 0.010 & 19 & & 0.019 \\
\hline & & 5 & & 7 & 9 & 0.0 & 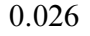 & 19 & 19 & & 19 & 19 & & 019 \\
\hline & Width & 527 & 7 & 35 & 0.671 & 0.671 & 611 & 0.506 & 0.601 & 6 & 0.610 & 37 & 35 & 0.503 \\
\hline \multirow[t]{4}{*}{20} & pver & 951 & 42 & 0.924 & 0.974 & 0.978 & 970 & 0.945 & 0.981 & 0.944 & 0.981 & & & 0.958 \\
\hline & wer & 14 & & 0.022 & 0.005 & 0.005 & 10 & 034 & 0.009 & 0.021 & 0.009 & & & 0.021 \\
\hline & & & & & 0.021 & & 19 & 021 & & & 011 & 21 & 62 & .021 \\
\hline & Width & 1 & & 77 & 66 & 0.566 & 34 & 38 & 18 & & 0.523 & 62 & 27 & 0.436 \\
\hline \multirow[t]{4}{*}{30} & Cover & 0.928 & 0.935 & .924 & 0.983 & 0.982 & 970 & 0.945 & 0.977 & 0.939 & 0.977 & 0.955 & 0.912 & 0.955 \\
\hline & bwer & 014 & 3 & .022 & 0.004 & 0.004 & 10 & 0.031 & 0.009 & 0.021 & 0.009 & .021 & 26 & 0.021 \\
\hline & Upper & 058 & & 0.054 & 0.014 & 0.014 & .020 & 0.024 & 0.014 & 0.039 & 0.014 & .024 & & 0.024 \\
\hline & Width & & & & & & & & 22 & & 25 & 74 & & 0.357 \\
\hline \multirow[t]{4}{*}{50} & Cover & & & & & & & & 77 & & 0.977 & 50 & & 0.950 \\
\hline & wer & 018 & & 23 & 03 & 0.005 & 09 & 0.032 & 0.009 & 0.024 & 0.009 & .024 & 26 & 0.024 \\
\hline & Upper & 337 & & 14 & 0.013 & 0.015 & 015 & 26 & 0.013 & 0.037 & 0.013 & .026 & & .026 \\
\hline & Width & 0.280 & 0.280 & 0.273 & 0.346 & 0.346 & .337 & 0.277 & 0.326 & 0.277 & 0.327 & 0.287 & 0.273 & 0.277 \\
\hline \multirow[t]{4}{*}{100} & Cover & 0.947 & & 0.947 & 0.986 & 0.985 & & 0.954 & 0.977 & & 0.980 & 0.958 & 0.943 & 0.958 \\
\hline & & & & & & & & & & & & 22 & & 0.022 \\
\hline & & & & & & & & & & & 0.008 & 20 & & .020 \\
\hline & Width & 0.197 & 0.197 & 0.194 & 0.242 & 0.242 & 0.238 & 0.196 & 0.230 & 0.196 & 0.231 & 0.201 & 0.194 & 0.196 \\
\hline
\end{tabular}

Table 7. The estimated coverage probabilities and average widths for lambda $=2$ and confidence coefficient 0.95

\begin{tabular}{|c|c|c|c|c|c|c|c|c|c|c|c|c|c|c|}
\hline $\mathrm{N}$ & & Wald & WaldB & Waldz & Wcc & WccB & Wccz & $\mathrm{S}$ & FT & VSS & RVS & Exact & Boot & Bayes \\
\hline \multirow[t]{4}{*}{5} & Cover & 0.923 & 0.923 & 0.806 & 0.964 & 0.964 & 0.850 & 0.958 & 0.954 & 0.954 & 0.954 & 0.973 & 0.767 & 0.940 \\
\hline & Lower & 0.008 & 0.008 & 0.069 & 0.005 & 0.006 & 051 & 0.030 & 016 & 0.016 & 016 & 016 & 085 & .030 \\
\hline & Upper & 068 & 068 & 125 & 0.030 & 0.030 & 099 & 0.011 & 0.030 & 0.030 & 0.030 & & .148 & .030 \\
\hline & Width & 938 & 938 & 752 & 072 & 1.072 & 845 & 876 & 980 & 0.876 & 0.977 & 10 & 760 & 868 \\
\hline \multirow[t]{4}{*}{10} & Cover & 0.955 & 0.950 & 0.902 & 0.978 & 0.976 & .932 & 0.951 & 0.972 & 0.948 & 0.972 & 0.965 & .880 & .965 \\
\hline & Lower & 013 & 0.011 & 0.033 & 0.006 & 0.005 & .025 & 0.033 & 0.013 & 0.020 & 0.013 & .020 & .042 & 0.020 \\
\hline & Upper & .032 & 039 & 0.064 & 0.016 & 0.019 & 12 & 0.016 & & & 0.016 & & 79 & 0.016 \\
\hline & Width & & & & 22 & & & 0.620 & & & 0.682 & & & 0.617 \\
\hline \multirow[t]{4}{*}{15} & Cover & 931 & & 15 & 0.972 & 0.970 & & 0.946 & & 0.941 & 0.962 & & 99 & 955 \\
\hline & 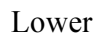 & 15 & & 29 & 0.006 & 0.007 & & 0.031 & & 0.023 & 0.015 & & & 023 \\
\hline & Upper & .054 & & 56 & 0.022 & 0.023 & & 0.022 & & & & & & 022 \\
\hline & Width & & & & 0.581 & & & 506 & & & & & & 505 \\
\hline \multirow[t]{4}{*}{20} & $\mathrm{Co}$ & & & & & & & & & & & & & 39 \\
\hline & Lo & & & & 1 & 0.009 & & 32 & & 0. & 0.0 & & & 32 \\
\hline & & & & & & & & 20 & & & 0.020 & & & .028 \\
\hline & Width & 0.444 & 0.444 & 421 & 0.499 & 0.499 & $J$ & 0.438 & 0.484 & 0.438 & 0.480 & & 0.421 & 0.437 \\
\hline \multirow[t]{4}{*}{30} & Cover & 949 & & 0.937 & 0.976 & 0.974 & & 0.962 & 0.969 & & 0.969 & & 29 & 954 \\
\hline & & & & & & & & & & & & & & \\
\hline & & & & & & & & 17 & & & 0. & & & 025 \\
\hline & & & & & & & & 58 & & & 0.391 & & & .357 \\
\hline \multirow{4}{*}{50} & Cover & 0.945 & 0.947 & 0.943 & 0.972 & 0.971 & & 0.953 & 0.969 & 0.951 & 0.969 & & 38 & 0.958 \\
\hline & Lower & & & & 0.011 & & & & & & 0.014 & & & .021 \\
\hline & & & & & & & & & & & & & & \\
\hline & & & & & & & & 77 & & & & & & 277 \\
\hline \multirow[t]{4}{*}{100} & & & & & 0.972 & & & & & & 0.970 & & & 0.951 \\
\hline & Lower & 021 & & .025 & 0.013 & 0.012 & & 0.030 & 0.015 & 0.026 & 0.015 & 0.026 & 0.026 & 0.026 \\
\hline & Upper & 0.033 & 0.030 & 0.030 & 0.015 & 0.016 & 17 & 0.022 & 0.015 & 0.027 & 0.015 & 0.022 & 0.033 & 0.022 \\
\hline & Width & 0.196 & 0.196 & 0.193 & 0.220 & 0.220 & 0.216 & 0.196 & 0.216 & 0.196 & 0.214 & 0.199 & 0.193 & 0.196 \\
\hline
\end{tabular}


Florence George and B.M. Golam Kibria / American Journal of Biostatistics 2 (2) (2011) 44-55

Table 8. The estimated coverage probabilities and average widths for lambda $=5$ and confidence coefficient 0.95

\begin{tabular}{|c|c|c|c|c|c|c|c|c|c|c|c|c|c|c|}
\hline $\mathrm{N}$ & & Wald & WaldB & Waldz & Wec & WccB & Wccz & $\mathrm{S}$ & FT & VSS & RVS & Exact & Boot & Bayes \\
\hline \multirow[t]{4}{*}{$\overline{5}$} & Cover & 0.947 & 0.944 & 0.832 & 0.953 & 0.953 & 0.858 & 0.944 & 0.965 & 0.939 & 0.965 & 956 & 0.812 & 0.956 \\
\hline & Lower & 014 & 012 & 070 & 0.009 & 0.009 & 059 & 0.035 & 0.014 & 0.023 & 0.014 & 023 & 079 & 0.023 \\
\hline & Upper & 38 & 44 & 098 & 038 & 0.038 & 083 & .021 & 021 & .038 & 0.021 & 021 & 109 & .021 \\
\hline & Width & 895 & 895 & 0.736 & 0.943 & 0.943 & .775 & 0.877 & 0.919 & 0.877 & 0.912 & .918 & .736 & 0.874 \\
\hline \multirow[t]{4}{*}{10} & Cover & 0.946 & 0.941 & 0.898 & 0.951 & 0.953 & 911 & 0.942 & 0.958 & 0.938 & 0.958 & .950 & .887 & 0.950 \\
\hline & Lower & 0.019 & 0.017 & 0.042 & 0.014 & 0.014 & 037 & 0.034 & .019 & 0.027 & 0.019 & & .046 & 0.027 \\
\hline & Upper & 5 & & 0 & 5 & 0. & 2 & 23 & & & 23 & & & 023 \\
\hline & Width & 626 & 26 & 0.574 & 658 & 0.658 & 3 & 0.620 & 49 & 20 & 0.644 & & 74 & 519 \\
\hline \multirow[t]{4}{*}{15} & Cover & 951 & 46 & 0.910 & 0.951 & 0.956 & 24 & 0.942 & 0.955 & 0.949 & 0.955 & & 0.902 & 0.949 \\
\hline & Lower & 0.014 & 0.016 & 0.032 & 0.014 & 0.012 & .027 & 0.030 & .017 & & 0.017 & & & 0.024 \\
\hline & Upper & 036 & & 0.058 & 0.036 & 0.032 & .049 & 0.028 & & & 0.028 & & 64 & 0.028 \\
\hline & Width & & & & & & & 6 & & & & & & 0.506 \\
\hline \multirow[t]{4}{*}{20} & Cover & 94 & & 921 & 0.955 & 0.956 & 933 & 0.948 & 8 & 0.9 & 0.958 & & & 53 \\
\hline & Lower & 018 & & 31 & 13 & 0.013 & & 27 & & & & & & 0.023 \\
\hline & Upper & .040 & 0.039 & 047 & 0.032 & 0.031 & 0.040 & 0.025 & 0.025 & 0.032 & 0.025 & & 0.051 & 0.025 \\
\hline & Width & & & & 462 & & 39 & 438 & 59 & & 55 & & & 438 \\
\hline \multirow[t]{4}{*}{30} & Cover & & & & & & & 58 & & & & & & 0.951 \\
\hline & Lower & 018 & & 0 & 0.014 & 0.0 & 66 & 0.026 & 0.018 & 0.026 & 0.023 & 26 & 32 & 0.026 \\
\hline & Upper & & & & & 0.0 & & 16 & & & 0.023 & & & 0.023 \\
\hline & Width & 0.359 & 0.359 & 0.347 & 0.377 & 0.377 & 0.364 & 0.358 & 0.374 & 0.358 & 0.371 & & 0.347 & 0.358 \\
\hline \multirow[t]{4}{*}{50} & Cover & 0.952 & & 0.943 & 0.963 & 0.963 & & 0.947 & 0.962 & & 0.962 & & 0.942 & 0.951 \\
\hline & Lower & & & & & & & & & & & & & \\
\hline & & & & & & 0 . & & & & & & & & 0.022 \\
\hline & Width & 0.278 & & 0.272 & 0.291 & 0.291 & 0.285 & 0.277 & 0.290 & 0.277 & 0.287 & 0.282 & 0.272 & 0.277 \\
\hline \multirow[t]{4}{*}{100} & Cover & 0.946 & 0.946 & 0.941 & 0.958 & 0.957 & 0.953 & 0.946 & 0.956 & 0.947 & 0.956 & 0.949 & 0.940 & 0.949 \\
\hline & Lower & 02 & & & 0.022 & 0.021 & & 0.031 & & 0.028 & & & & 0.028 \\
\hline & Upper & & & & 0.021 & 0.022 & & 0.023 & & & & & & 0.023 \\
\hline & Width & 0.196 & 0.196 & 0.193 & 0.206 & 0.206 & 0.203 & 0.196 & 0.205 & 0.196 & 0.203 & 0.198 & 0.193 & 0.19 \\
\hline
\end{tabular}

Table 9. The estimated coverage probabilities and average widths for lambda $=10$ and confidence coefficient 0.95

\begin{tabular}{|c|c|c|c|c|c|c|c|c|c|c|c|c|c|c|}
\hline $\mathrm{N}$ & & Wald & WaldB & Waldz & $\mathrm{Wcc}$ & WccB & Wccz & $\mathrm{S}$ & FT & VSS & RVS & Exact & Boot & Bayes \\
\hline \multirow[t]{4}{*}{5} & Cover & 0.945 & 0.941 & 0.834 & 0.950 & 0.948 & 0.849 & 0.935 & 0.954 & 0.936 & 0.954 & 0.945 & 0.819 & 0.945 \\
\hline & Lower & 0.018 & 0.017 & 072 & 0.013 & 0.014 & 064 & 0.037 & .018 & 0.027 & 018 & 27 & 779 & .027 \\
\hline & Upper & .037 & 0.042 & 094 & 0.037 & 0.038 & 087 & 0.028 & 0.028 & 0.037 & 0.028 & & 02 & .028 \\
\hline & Width & 885 & 0.885 & 729 & 0.908 & 0.908 & 747 & 877 & 98 & 0.877 & 0.893 & 97 & & .875 \\
\hline \multirow[t]{4}{*}{10} & Cover & 0.946 & 0.948 & 0.897 & 0.958 & 0.955 & 0.908 & 0.953 & 0.960 & 0.952 & 0.957 & 957 & 389 & .957 \\
\hline & Lower & 017 & 0.017 & .043 & 0.014 & 0.015 & 039 & 0.025 & 0.017 & 0.020 & 0.020 & & & 0.020 \\
\hline & Upper & 0.037 & 035 & 060 & 0.028 & 0.029 & 53 & 0.023 & & 0.028 & .023 & & & .023 \\
\hline & Width & 0 & & & & & & 0 & & & & & & 619 \\
\hline \multirow[t]{4}{*}{15} & Cover & 95 & & 16 & 0.953 & 0. & 4 & 6 & & 1 & 955 & & & 951 \\
\hline & $\mathrm{L}$ & 16 & & & 0.016 & 0.016 & & 24 & & 0.024 & .020 & & & 024 \\
\hline & Upper & 0.031 & & 9 & 0.031 & 0.030 & & 20 & & 0.025 & 0.025 & & & .025 \\
\hline & Width & & & & & & & & & & 516 & & & 506 \\
\hline \multirow[t]{4}{*}{20} & Cov & & & & & & & & & & & & & 957 \\
\hline & Lov & 0 & & & & & & 5 & & & 0.019 & & & .022 \\
\hline & $\mathrm{U}$ & & & & 25 & & & & & 5 & .021 & & & .021 \\
\hline & Width & 0.439 & 39 & 419 & 0.450 & 0.450 & 0.430 & 438 & 0.449 & 0.438 & 0.446 & & 0.419 & 0.438 \\
\hline \multirow[t]{4}{*}{30} & Cover & 945 & & & 0.948 & & & 943 & 0.947 & 0.943 & 0.947 & & & 945 \\
\hline & & & & & & & & & & & & & & 29 \\
\hline & & & & & & & & & & & 26 & & & 0.026 \\
\hline & Width & & & & & & & & & & 365 & & & 0.358 \\
\hline \multirow[t]{4}{*}{50} & Cover & 0.947 & 0.947 & 0.937 & 0.954 & 0.952 & 0.943 & 0.945 & 0.953 & 0.948 & 0.953 & & 0.937 & 0.950 \\
\hline & Lower & & & & & & & & & & & & & 0.025 \\
\hline & & & & & & & & & & & & & & .025 \\
\hline & Width & & & & & & & & & & 82 & & & 277 \\
\hline \multirow[t]{4}{*}{100} & Cover & & & & 0.956 & & & & & & 0.955 & & & 0.949 \\
\hline & Lower & 0025 & 0.024 & 0 & 0.021 & 0.021 & 0.025 & 0.031 & & 0.029 & 0.023 & & 0.028 & 0.029 \\
\hline & Upper & 0.025 & 0.026 & 0.029 & 0.023 & 0.023 & 0.027 & 0.023 & 22 & 23 & 0.022 & 22 & 0.029 & 0.023 \\
\hline & Width & 0.196 & 0.196 & 0.194 & 0.201 & 0.201 & 0.198 & 0.196 & 0.201 & 0.196 & 0.200 & 0.198 & 0.194 & 0.196 \\
\hline
\end{tabular}




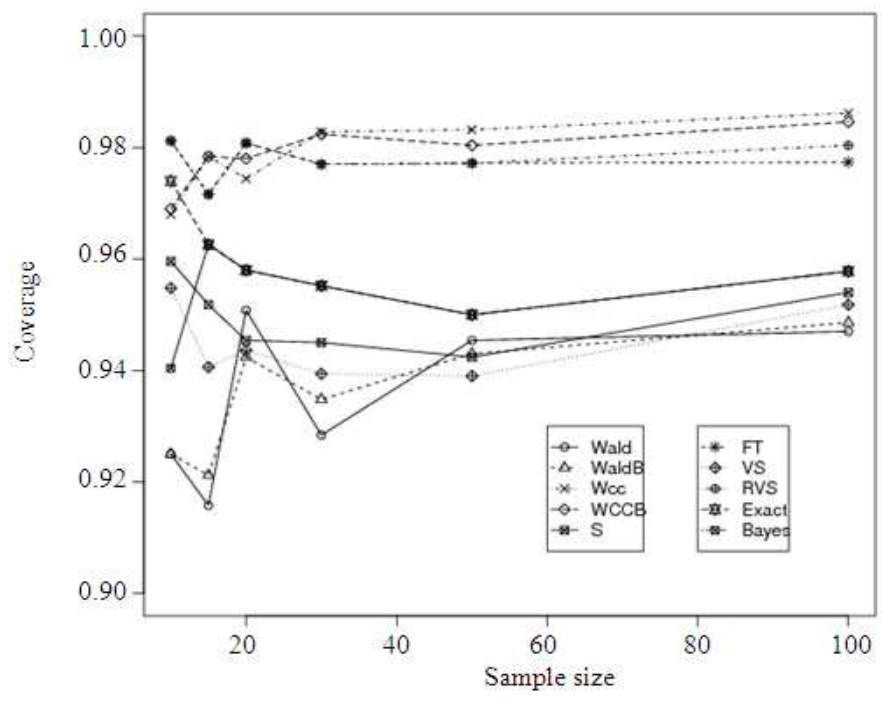

Fig. 1. Coverage vs $n, \lambda=1$, confidence coefficient $=0.95$

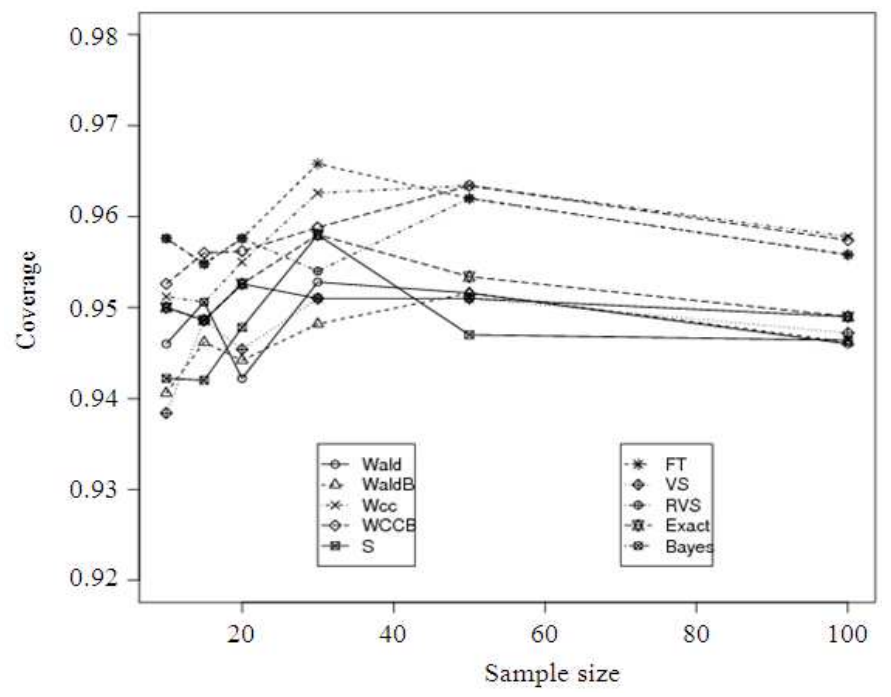

Fig. 2. Coverage vs $n, \lambda=5$, confidence coefficient $=0.95$

The coverage probability vs sample size for $\lambda=1$ and $\lambda$ $=5$ are presented in Fig. 1 and 2 respectively. The average width vs sample size for $\lambda=1$ and $\lambda=5$ are presented in Fig. 3 and 4 respectively. The coverage probability vs $\lambda$ and average width vs $\lambda$ for $n=30$ are presented in Fig. 5 and 6 respectively.

\section{RESULTS}

From Table 2-9 and Fig. 1-6, we can see a general pattern is that as the sample size increase the nominal sizes for all estimators also increase and converge to 0.90 (Table 2-5) and 0.95 (Table 6-9). Results also indicated that there are not much fundamental differences among the interval estimators when both sample and SNR are large. However, they performed differently for small sample size and small SNR. For all sample sizes, the exact method performed the best when the SNR is large in the sense of attaining nominal size and smaller width. It also performed the best in the sense of the symmetry of lower and upper error rates. For small samples, the Waldz, WccZ, bootstrap methods are performing poorly. A possible 
explanation for this is the sampling distribution of the bootstrapped statistics is frequently not symmetric. Thus, the size $(\alpha)$ of the critical region for bootstrap $t$ is not actually divided equally. In fact, the main contribution comes from the upper tail of the test statistic. We also note that the bootstrap version of Wald (named as WaldB) and Wcc (named as WccB) performed better than the corresponding Wald and Wcc methods. However, the performnace of WaldB is better than WccB.

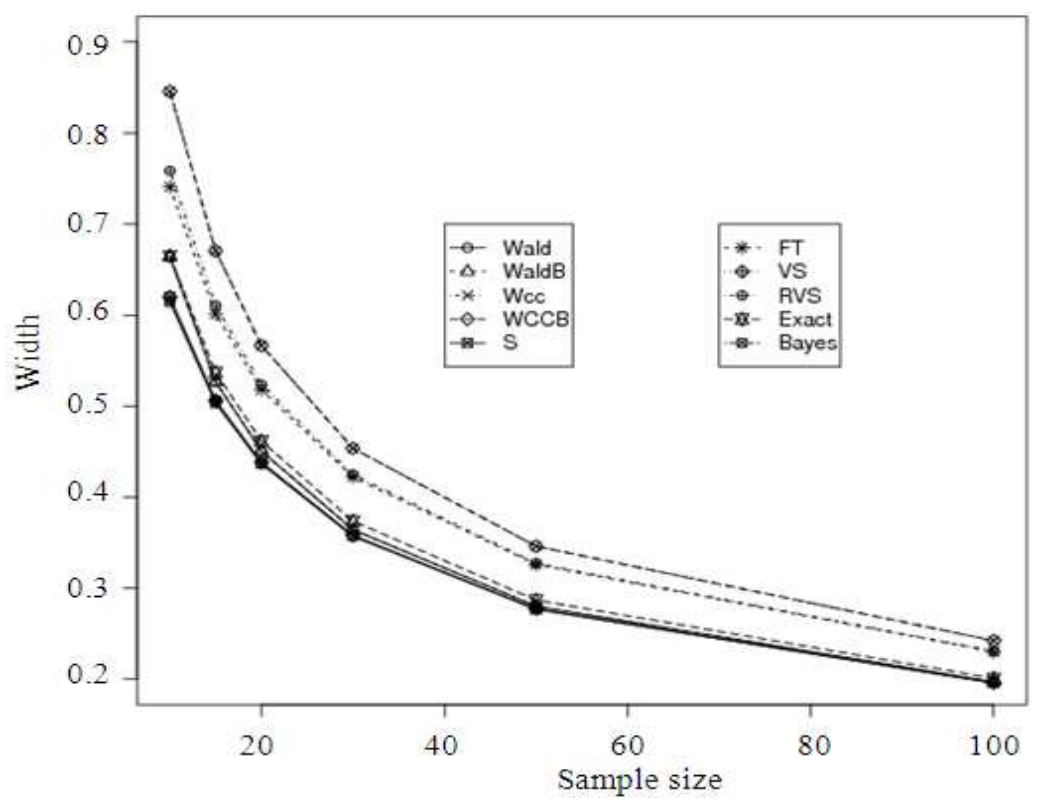

Fig. 3. Width vs $\mathrm{n}, \lambda=1$, confidence coefficient $=0.95$

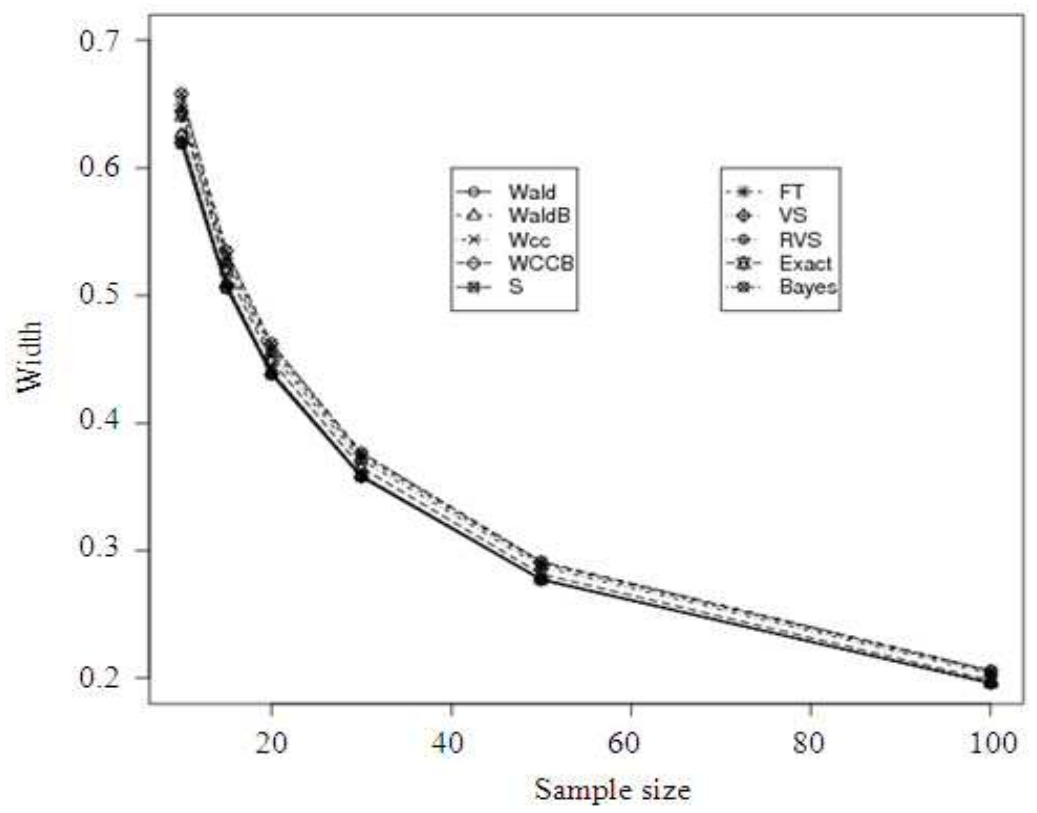

Fig. 4. Width vs $n, \lambda=5$, confidence coefficient $=0.95$ 


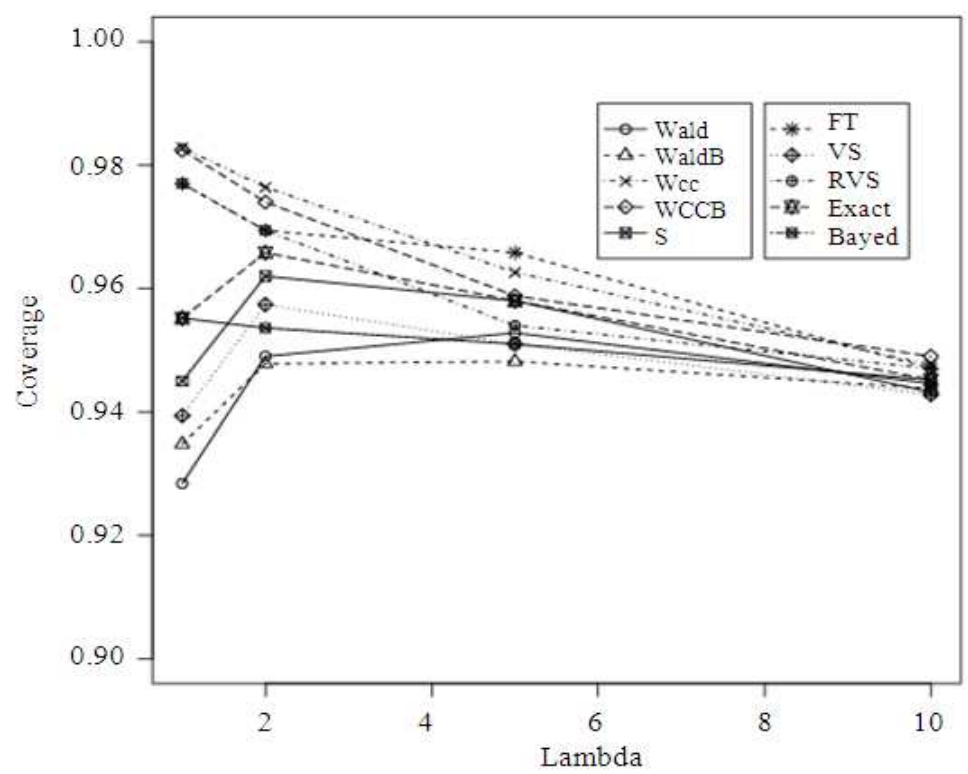

Fig. 5. Coverage vs $\lambda, n=30$, confidence coefficient $=0.95$

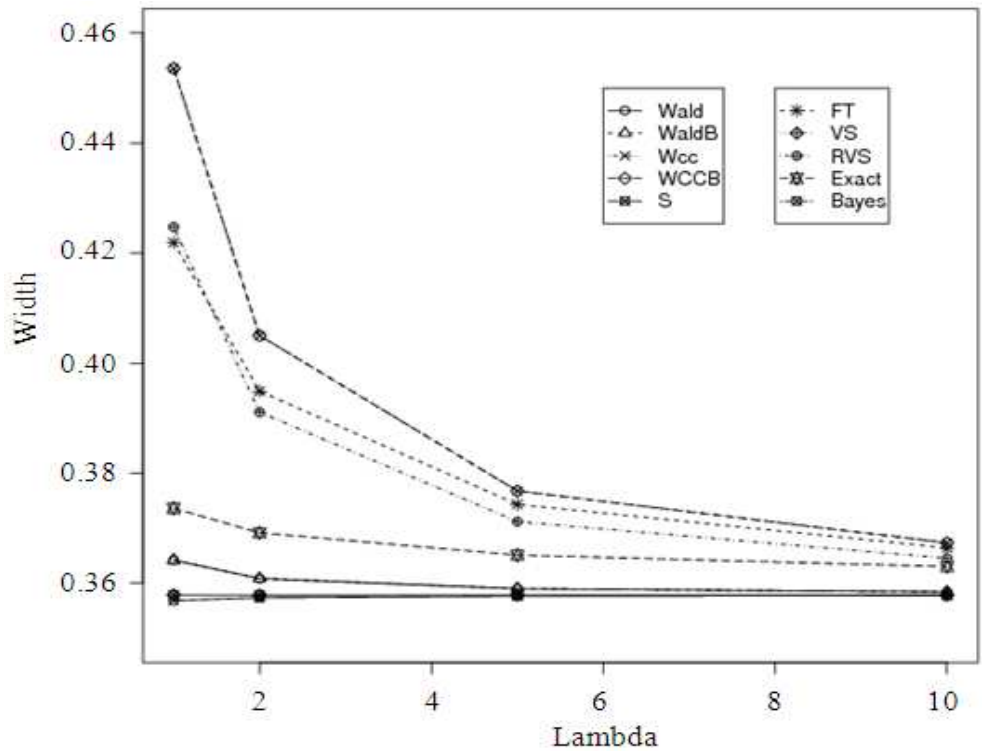

Fig. 6. Width vs $\lambda, n=30$, confidence coefficient $=0.95$

\section{DISCUSSION}

From Table 2-9 and Fig. 1-6, we can see a general pattern is that as the sample size increase the nominal sizes for all estimators also increase and converge to 0.90 (Table 1-4) and 0.95 (Table 6-9). Results also indicated that there are not much fundamental differences among the interval estimators when both sample and SNR are large. However, they performed differently for small sample size and small SNR. For all sample sizes, the exact method performed the best when the SNR is large in the sense of attaining nominal size and smaller width. It also performed the best in the sense of the symmetry of lower and upper error rates. For small samples, the Waldz, WccZ, bootstrap methods are performing poorly. A possible explanation for this is the sampling distribution of the bootstrapped statistics is frequently not symmetric. 
Thus, the size $(\alpha)$ of the critical region for bootstrap $t$ is not actually divided equally. In fact, the main contribution comes from the upper tail of the test statistic. We also note that the bootstrap version of Wald (named as WaldB) and Wcc (named as WccB) performed better than the corresponding Wald and Wcc methods. However, the performance of WaldB is better than WccB.

\section{CONCLUSION}

This study considered thirteen interval estimators for estimating the population Signal to Noise Ratio (SNR) for a Poisson distribution. Coverage probability, the symmetry of lower and upper error rates and average width are considered as a criterion of a good estimator. We observed that the performance of the estimators depend on the sample size and the values of the SNR. Overall, most of the proposed intervals except methods Wald, Waldz and bootstrap are performing well in the sense of attaining nominal size. The exact method performed the best followed by VSS, Wald B and Bayes in the sense of attaining nominal size and shorter width when the SNR is large. We believe that the findings of the study will be useful for the researcher that is mentioned in the introduction.

\section{REFERENCES}

Agresti, A. and B.A. Coull, 1998. Approximate is better than "exact" for interval estimation of binomial proportions. Am. Stat., 52: 119-126. DOI: 10.2307/2685469

Anscombe, F.J., 1948. The transformation of poisson, binomial and negative-binomial data. Biometrika, 35: 246-254. DOI: $10.2307 / 2332343$

Banik, S. and B.M.G. Kibria, 2010. Comparison of some parametric and nonparametric type one sample confidence intervals for estimating the mean of a positively skewed distribution. Commun. Stat. Simulat. Comput., 39: 361-389. DOI: 10.1080/03610910903474530

Banik, S. and B.M.G. Kibria, 2011. Estimating the population coefficient of variation by confidence intervals. Commun. Stat. Simulat. Comput., 40: 1236-1261. DOI: 10.1080/03610918.2011.568151

Barker, L., 2002. A Comparison of nine confidence intervals for a poisson parameter when the expected number of events is 5. Am. Stat., 56: 85-89.

Bartlett, M.S., 1947. The use of transformations. Biometrics, 13: 39-52.

Cai, T.T., 2005. One-sided confidence intervals in discrete distributions. J. Stat. Plann. Inform., 131: 6368. DOI: 10.1016/j.jspi.2004.01.005

Efron, B and R.J. Tibshirani, 1993. An Introduction to the Bootstrap. 1st Edn., Chapman and Hall, New York, ISBN-10: 0412042312, pp: 456.
Efron, B., 1979. Bootstrap methods: Another look at the Jackknife. Annals Stat., 7: 1-26.

Efron, B., 1987. Better bootstrap confidence intervals. J. Am. Stat. Assoc., 82: 171-185.

Freeman, M.F. and J.W. Tukey, 1950. Transformations related to the angular and the square root. Annals Math. Stat., 21: 607-611.

Garwood, F., 1936. Fiducial Limits for the poisson distribution. Biometrika, 28: 437-442. DOI: 10.1093/biomet/28.3-4.437

Jitendra, R.R., 2009. Multi-Sensor Data Fusion: Theory and Practice. 1st Edn., CRC Press, Boca Raton, ISBN-10: 1439800030, pp: 534.

John, C.R., 2007. The Image Processing Handbook. 6th Edn., CRC Press, ISBN-10: 1439840458, pp: 867.

Kelley, K., 2007. Sample size planning for the coefficient of variation from the accuracy in parameter estimation approach. Behav. Res. Methods, 39: 755-766. PMID: 18183888

Koopmans, L.H., D.B. Owen and J.I. Rosenblatt, 1964. Confidence intervals for the coefficient of variation for the normal and log normal distributions. Biometrika Trust, 51: 25-32. DOI: 10.1093/biomet/51.1-2.25

Lee, H.C., 2009. Introduction to Color Imaging Science. 1st Edn., Cambridge University Press, New York, ISBN-10: 0521103134, pp: 716.

McGibney, G. and M.R. Smith, 1993. An unbiased signal-to-noise ratio measure for magnetic resonance images. Med. Phys., 20: 1077-1078. PMID: 8413015

McKay, A.T., 1932. Distribution of the coefficient of variation and the extended $t$ distribution. J. Royal Stat. Soc., 95: 695-698.

Miller, G.E., 1991. Asymptotic test statistics for coefficients of variation. Commun. Stat. Theory Methods, 20: 33513363. DOI: 10.1080/03610929108830707

Sharma, K.K. and H. Krishna, 1994. Asymptotic sampling distribution of inverse coefficient-ofvariation and its applications. IEEE Trans. Reliab., 43: 630-633. DOI: 10.1109/24.370217

Tania, S., 2008. Image Fusion: Algorithms and Applications. 1st Edn., Academic Press, Amsterdam, ISBN-10: 0123725291, pp: 520.

Vangel, M.G., 1996. Confidence intervals for a normal coefficient of variation. Am. Stat., 15: 21-26.

Visintainer, P.F. and N. Tejani, 1998. Understanding and using confidence intervals in clinical research. J. Maternal-Fetal Med., 7: 201-2. PMID: 9730488

Willkinson, M.H.F. and F. Schut, 1998. Digital Image Analysis of Microbes: Imaging, Morphometry, Fluorometry and Motility Techniques and Applications. 1st Edn., John Wiley and Sons, Chichester, ISBN-10: 0471974404, pp: 578.

Wilson, E.B., 1927. Probable inference, the law of succession and statistical inference. J. Am. Stat. Assoc., 22: 209-212. DOI: 10.2307/2276774 